\title{
Chemical Modification of Si Nanowires for Bioconjugation
}

\author{
Woo Kyung Cho, Young Hwan Jung, Kyung-Bok Lee, Hyung Ju Park, \\ Yongseong Kim, 'Dongmok Whang, \\ Department of Chemistry, KAIST. Daejeon 305-70I. Korea. "E-mail: ischoigkaistack kr \\ "Division of Chemistry and Chemical Engineering, Kyzhynam Univensity, Masan. Gyeongham 637-701. Korea \\ "School of Advanced Materials Science and Engineering. Sungkyungkwan University: Stwon 440-746. Korea \\ Received October 4,2005
}

Key Words : Si nanowires, Surface modification, Self-assembled monolayers, Biotinylation, Biotin-streptavidin interactions

One-dimensional nanomaterials, such as nanoubes and nanowires, have been investigated in detail for numerous applications due to their extraordinary optical, mechanical, and electrical properties. 'In the context of both processing and properties of nanowires (.NWs), a detailed understanding of their surface chemistry is required to meet the technological applications of NWs. For example, the chemical and electronic stability of the surlace of NWs is particularly important for applications including nanowire-based logic elements and chemical and biological sensors, which require direct interfacing with their surrounding environment." While there have been many elforts to chemical modifications of carbon nanotubes, ${ }^{3}$ there have been a few reports on chemical modilications of NWs. ${ }^{4}$

Silicon nanowires ( $\mathrm{SiNWs}$ ) have recently attracted a great deal of atention because silicon (Si) is of techrological importance in microclectronics. Many successful synthetic strategies have now been developed to obtain bulk quantities of SiNWs using both gas-phase and condensed-phase techniques with or without metal catalysts. The chemical nature of the Si surlace has also been studied in detail. Si foms a very stable oxide and can be chemically passivated with a number of organic species. The reaction mechanisms have extensively been investigated for solution-phase and vapor-phase oxidation, metallization, nitridation, and organic monolayer-based passivation of both $\mathrm{Si}$ surfaces of singlecrystal substrates and surfaces of porous Si." Therefore, SiNWs are a very good candidate for studying chemical modilications of NW surfaces, and in this Note we report the chemical modification of SiNWs by a combination of the formation of covalently bonded, organic monolayers on the surface of $\mathrm{Si}$ NW $\mathrm{W}$ and successive surface organic reactions.

Boron-doped, single-crystal SiNWs were synthesized by gold nanocluster-calalyzed chemical vapor deposition with $\mathrm{Sill} \mathrm{H}_{4} / \mathrm{B}_{2} \mathrm{l}_{6}(1000: 1$ or $4000: \mathrm{l})$ as a vapor-phase reactant by following the reported procedure. ${ }^{54}$ The average diameter of SiNWs was $\sim 40 \mathrm{~nm}$ and the length of SiNWs was 3-6 km (Figure 1). The gradual bending of SiNWs may be due to elastic strains on the very small diameter of the wires.

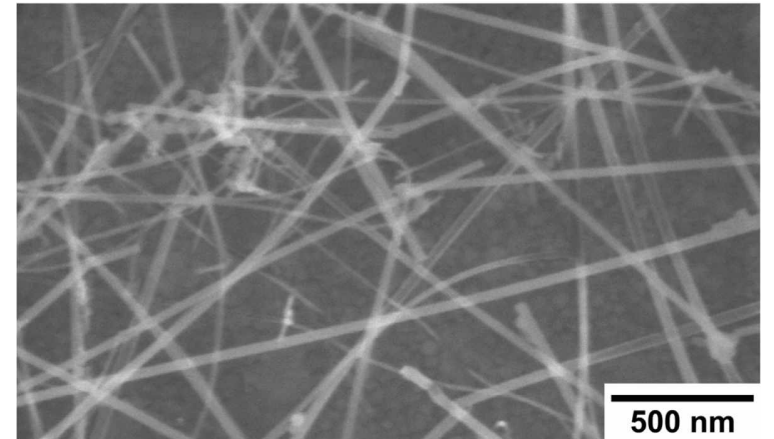

Figure 1. FE-SEM inage of synthesized boron-doped Si nanowires.

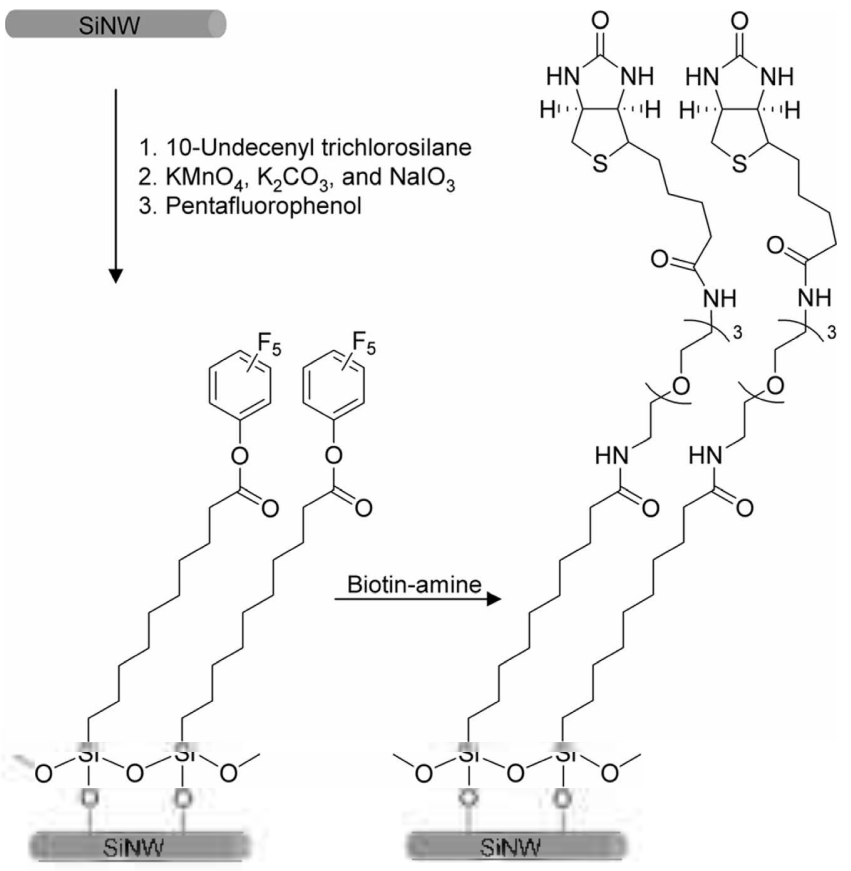

Scheme 1. Surface modilication of horon-doped Si nanowires (SiNWs). 


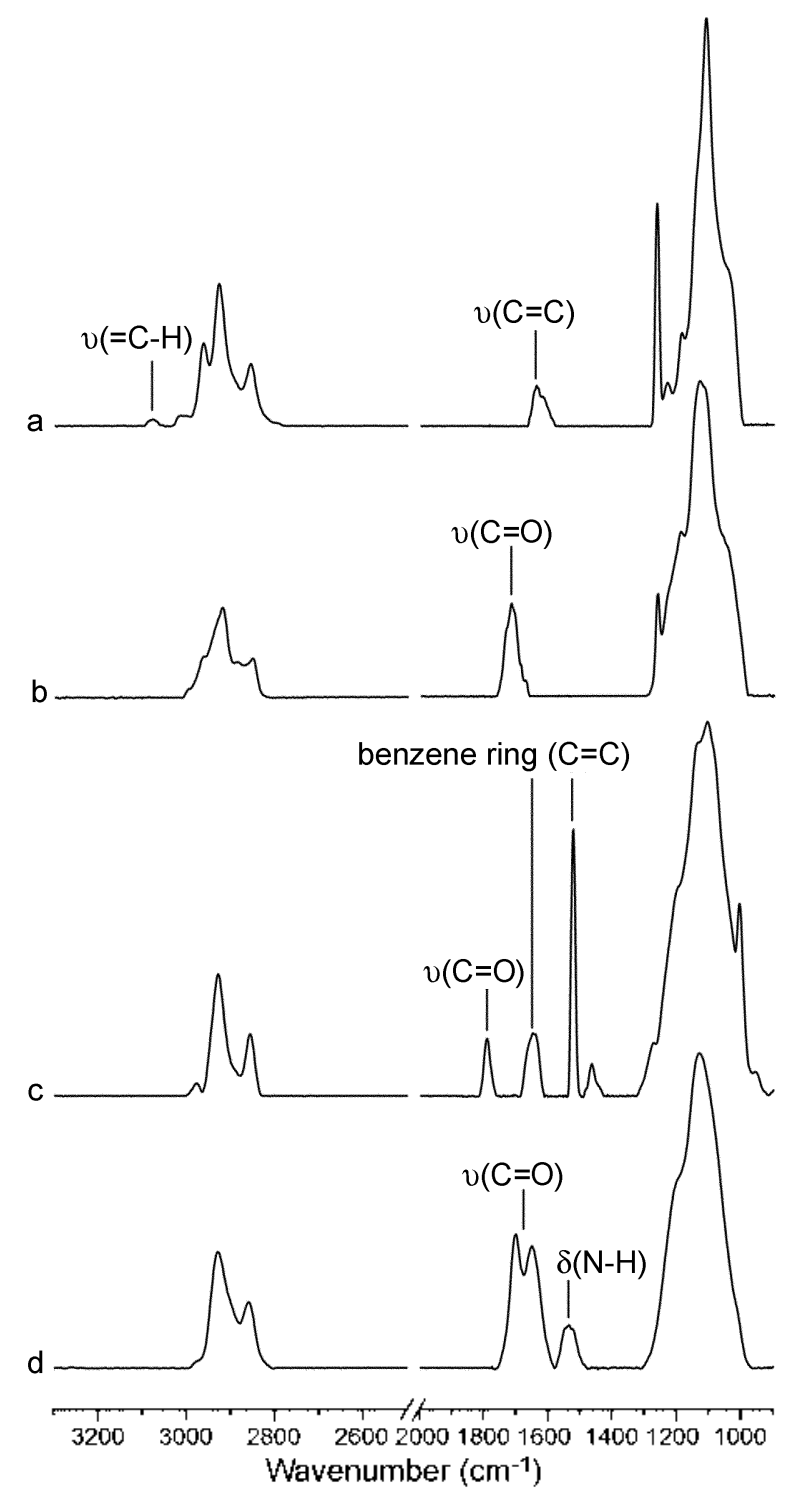

Figure 2. PIERS spectra of SiNWs at each step: (a) formation of SAMs of 10-undecenyl trichlorosilane, (b) oxidation to carboxylic acid groups, (c) PFP activation, and (d) amide coupling with biotinamine.

Scheme 1 briefly depicts our approach to the chemical modification of the surface of SiNWs. We applied the "common intermediate method" to chemically modify SiNWs because the method enables us to introduce functionalities onto SiNWs without any cumbersome synthesis of compounds of interest. Self-assembled monolayers (SAMs) of 10-undecenyl trichlorosilane were formed on the surface of SiNWs. The terminal vinyl groups on the surface of SiNWs were oxidized to carboxylic acid groups by $\mathrm{KMnO}_{4}, \mathrm{~K}_{2} \mathrm{CO}_{3}$ and $\mathrm{NaIO}_{3}{ }^{{ }^{*}}$ The carboxylic acid groups were then activated with $N$-(3-dimethylaminopropyl)- $N^{\prime}$-ethylcarbodimide hydrochloride (EDC) and pentafluorophenol (PFP)." The formation of the SAMs on SiNWs and the successive reactions were confirmed by polarized infrared external reflectance spectroscopy (PIERS). The IR spectrum of the vinyl-terminated SAMs of 10-undecenyl trichlorosilane showed characteristic peaks at $3078 \mathrm{~cm}{ }^{1}$ (=C-H stretehing) and $1639 \mathrm{~cm}^{3}(\mathrm{C}=\mathrm{C}$ stretching) (Figure 2a). After the oxidation of the terminal vinyl groups to carboxylic acid groups, the IR peaks from the vinyl groups disappeared and a new peak from $\mathrm{C}=O$ stretehing was observed at $1718 \mathrm{~cm}^{3}$ (Figure 2b). The sucecssful PFP-activation was confirmed by characteristic IR peaks at $1650 \mathrm{~cm}^{1}$ and $1523 \mathrm{~cm}^{1}$ (benzene ring, $\mathrm{C}=\mathrm{C}$ stretehing; Figure 2c). After the PFP activation, any molecules with amine functional groups ean be anchored onto the surface of SiNWs through an amide bond. Because our ultimate goal of the study was to achieve bio-inspired, directed assembly of electronic nanocircuits made of semiconductor NWs and to construct celectronic devices or biological sensors, we seleeted amine-containing biotin as a model of amine compounds in order to investigate the feasibility of our approach to bicconjugation. In addition, the biotin-(strept)avidin interactions were recently utilized to make the connection between metal gold nanorods or nanowires. ${ }^{10} \mathrm{To}$ attach a biotin moicty onto SiNWs, the PFP-activated SiNWs were immersed in an ethanol solution of (+)-biotinyl-3,6,9-trioxaundecancdiamine ("biotin-amine" $y^{\mathrm{x}+.9 .11}$ for $30 \mathrm{~min}$ and washed thoroughly with ethanol several times. After the attachment of biotin-amine, we observed new IR peaks from N-H bending $\left(1547 \mathrm{~cm}^{1}\right)$ and $\mathrm{C}=\mathrm{O}$ stretching $\left(1650 \mathrm{~cm}{ }^{\prime}\right.$ and $\left.1701 \mathrm{~cm}^{1}\right)$ (Figure $2 \mathrm{~d}$ ). The stretching mode of $\mathrm{C}=\mathrm{O}$ amide bond of alkyl chains was shown at $1650 \mathrm{~cm}^{3}$.

The chemical modification and subsequent attachment of biotin onto SiNWs was also verified by fluoreseence confocal microscopy after a complexation of the biotin-attached SiNWs with rhodamine (TRITC)-conjugated streptavidin. The biotin-avidin interactions are one of the strongest biological interactions $\left(\mathrm{K}_{\mathrm{T}}=10^{15} \mathrm{M}^{1}\right)$ and stable over a broad range of $\mathrm{pH}$. Streptavidin has two pairs of binding sites on opposite sides, and therefore the multivalency of streptavidin could be used for the directed assembly of SiNWs in solution as well as the directed depesition of SiNWs on biotin-functionalized surfaces. We functionalized "as grown" SiNWs on a silicon wafer without detachment of SiNWs from the wafer (see the Experimental Section): when we detached SiNWs from the wafer and then functionalized free-standing SiNWs in solution, the sonication processes greatly shortened SiNWs. In addition, SiNWs had a tendency for aggregation in aqueous phase. After the modification, SiNWs were washed with phosphate-buffered saline (PBS, $\mathrm{pH} 7.4)$ containing $0.1 \%(\mathrm{w} / \mathrm{v})$ bovine serum albumin (BSA) and $0.02 \%(\mathrm{v} / \mathrm{v})$ Tween 20 in order to minimize nonbiospecific adsorption of streptavidin, and complexed with TRITC-conjugated streptavidin in PBS containing BSA and Tween 20 for $1 \mathrm{~h}$. Figures $3 \mathrm{a}$ and $\mathrm{b}$ show the fluorescence and optical micrographs of SiNWs before and after the complexation with TRITC-conjugated streptavidin, respectively. We did not observe any fluorescence from the biotinattached SiNWs before the complexation (Figure 3a), but observed a clear red fluorescence after the complexation (Figure 3b), which clearly confirms the successful attachment of biotin onto SiNWs. ${ }^{2}$ 

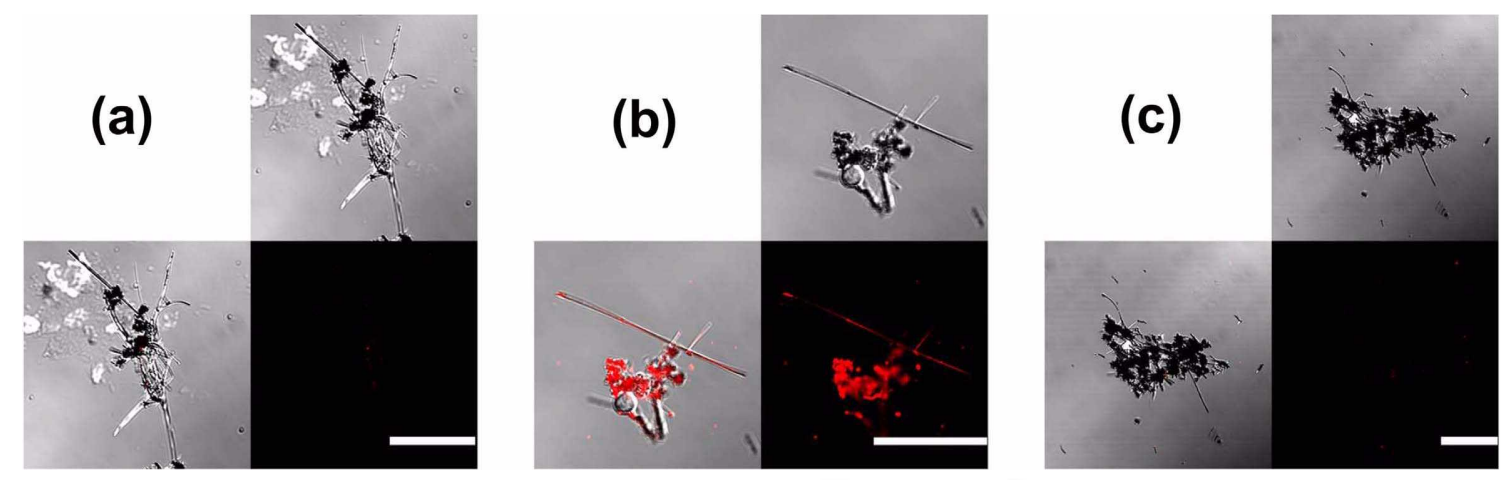

Figure 3. Fluorescestee and optical micrographs of biolin-atlached SiNWs (a) before and (b) aller a complexation with TRITC-conjugaled streptavidin, (c) Fluorescence and optical micrographs of biotin-altached SiNWs atter incubation with TRITC-conjugaled streptavidin that was pre-treated with soluble biotin. The scale bar is $3 \mu \mathrm{mm}$.

Although we used BSA and Tween 20 to minimize the non-biospecific adsorption of streplavidin, we did not confirm that the obseryed red fluoresene resulted from bisspecilic interactions between biotin and streptavidin. To prove that the red fluorescence was derived from the specilic biotin-streptavidin interactions, a control experiment was performed: TRITC-conjugated streptavidin was pre-saturated with soluble biotin and hence the binding sites of the streptavidin were blocked. The biotin-atlached SiNWs were then immersed in a PBS solution containing the pre-treated streptavidin. We did not observe any red fluorescene from the SiNWs (Figure 3e). Therefore, we believe that the observed red florescence resulted from the specific biotinstreptavidin interactions.

In summary, we demonstrated that SiNWs were chemically modified to present biologically active biotin, using a combination of the formation of vinyl-terminated SAMs on SiNWs and the sucessive surface reactions. The procedure described herein could be generalized to the attachment of other biologically active molecules onto SiNWs ("bioconjugation"), and the control over surlace chemistry of SiNWS for bioconjugation would be a key requirement for many applications, such as nano-electronic devices, biological sensors, and nanoscale superstructures.

\section{Experimental Section}

Materials. 10-Undecenyl trichlorosilane (Gelest, Inc.), absolute toluene (99.9\%, Merck), polassium permanganate $\left(\mathrm{KMnO}_{4}, 99+\%\right.$, Aldrich $)$, sodium periodale $\left(\mathrm{NaIO}_{4}, 99 \%\right.$, Nldrich), potassium carbonate $\left(\mathrm{K}_{2} \mathrm{CO}_{3}, 99+\%\right.$, Nldrich), sodium hydrogensulfite ( $\mathrm{NaIISO}_{3}$, A.C.S. reagent, Aldrich), hydrochloric acid ( $\mathrm{HCl}, 35 \%$, Junsei), $N$-(3-dimethylaminopropyl)- $N$-cthylcarbodiinide hydrochloride (TDC, commercial grade, Aldrich), pentalluorophenol (PFP, 99 $\%$, Aldrich), absolute ethanol (99.8\%, Merck), (+)-biotinyl3,6,9-rioxaundecanedianine (Piere), phosphate bulfered saline (PBS, pH 7.4, Sigma), bovine serum albumin (BSA, Sigma), Tween 20 (Aldrich), and rhodamine (TRITC)conjugated streptavidin (Pieree) were used as received. Ultrapure waler $(18.3 \mathrm{M} \Omega / \mathrm{cm})$ from the Iluman Ultra Pure
System (Human Corp., Korea) was used.

Synthesis of Boron-Doped Si Nanowires (SiNWs). Boron-doped, single-crystal silicon nanowires (SiNWs) were synthesized by gold nanocluster-catalyzed chemical vapor deposition. ${ }^{5 \mathrm{~K}} \mathrm{At}$ first, growth substrates were prepared by depositing $0.1 \%$ poly- $l$-lysine (Ted Pella) and 30-nm gold nanoclusters (Ted Pella) on oxidized silicon wafers. The negatively charged nanoclusters stuck to the positively charged poly- $l$-lysine. The substrates were eleanced in an oxygen plasma cleaner, and then placed in a cuartz. reactor at the center of the furnace. The reactor was evacuated to less than $100 \mathrm{mTorr}$ and heated to $460^{\circ} \mathrm{C}$ under $\mathrm{Ar}$ nlow, and then SiNWs were grown for 20-30 min with a $15-30 \mathrm{secm}$ flow of $\mathrm{SHII}_{2} / \mathrm{B}_{2} \mathrm{Il}_{6}$, ratio of either $1000: 1$ or $4000: 1(10 \%$ in $\mathrm{He}$ ).

Surface Modification of SiNWs. All processes were perfomed with SiNWs on a Si waler as synthesized. Prior to the formation of $\mathrm{S} \Lambda \mathrm{Ms}$, SiNWs were oxidized by an oxygen plasma cleaner (IIarrick PD)C-002, medium setting) for 1 min to generate -OII groups. Vinyl-terminated SAMs were formed by immersing the oxidized SiNWs in a $0.5 \%$ toluene solution of 10-undecenyl trichlorosilane for $30 \mathrm{~min}$. After the formation of SAMs, the SiNWS were washed carefully with toluene several times. The terminal vinyl groups were then oxidized to carboxylic acid by inmersing the SiNWs in a solution of $0.5 \mathrm{mM}$ of $\mathrm{KMnO}_{4}, 19.5 \mathrm{mM}$ of $\mathrm{NaJO}_{4}$, and 1.8 $\mathrm{mM}$ of $\mathrm{K}_{2} \mathrm{CO}_{3}$ for $24 \mathrm{~h}$. ${ }^{8}$ The $\mathrm{SiNW}$ s were removed from the oxidant and washed carefully with NaIISO: $(0.3 \mathrm{M})$, waler, $0.1 \mathrm{~N} \mathrm{HCl}$, waler, and ethanol. ${ }^{8_{1}}$ Carboxylic acid-terminated surfaces were activated by immersing the SiNWs in an ethanol solution of EDC $(0.1 \mathrm{M})$ and PFP $(0.2 \mathrm{M})$ for 30 min. ${ }^{8.4}$ The PFP-activaled SiNWs were washed carefully with ethanol several times. The sample was then immersed in a solution of biotin-amine (10 $\mathrm{mM}$ in cthanol) for $30 \mathrm{~min}$ and washed carefully with ethanol several times. By sonication, the SiNWs were then separated from the Si wafer and dispersed in ethanol. The dispersed SiNWs were spun off by using centrifugation and washed with phosphatebulfered saline (PBS, pll 7.4) containing $0.1 \%(w / v)$ BSA and $0.02 \%(\mathrm{v} / \mathrm{v})$ Tween 20 several times. $\Lambda$ solution of TRITC-conjugated streptavidin $(0.1 \mathrm{mg} / \mathrm{mL})$ in PBS 
containing $0.1 \% \mathrm{BSA}$ and $0.02 \%$ Tween 20 was then injected into a micro-centrifuge tube containing the biotinattached SiNWs. After $60 \mathrm{~min}$, the SiNWs were spun off by centrifugation, washed several times with PBS containing $0.1 \%$ BSA and $0.02 \%$ Tween 20 , and dispersed in PBS solution containing $0.1 \%$ BSA and $0.02 \%$ Tween 20 . Fluorescence confocal microscopy was used to examine the streptavidin-bound SiNWs. As a control experiment, TRITC-conjugated streptavidin (1 $\mathrm{mg} ; 10 \mathrm{mM}$ ) was incubated with soluble biotin $(40 \mathrm{mM})$ in PBS for $60 \mathrm{~min}$. The biotin-attached SiNWs were then immersed in a PBS solution (containing $0.1 \% \mathrm{BSA}$ and $0.02 \%$ Tween 20 ) of the pre-treated TRITC-conjugated streptavidin for $60 \mathrm{~min}$ and washed with $\mathrm{PBS}$ containing $0.1 \% \mathrm{BSA}$ and $0.02 \%$ Tween.

Instrumentation. Field-emission scanning electron microscopy (FE-SEM) image of boron-doped SiNWs was obtained with a scanning electron microscope (FEI, Sirion-400). All fluorescence and optical images were obtained with an LMS 510 laser scanning confocal microscope (Carl Zeiss, Germany). PIERS spectra were recorded on a Thermo Nicolet Nexus Fourier transform infrared spectrometer in a $\mathrm{SAGA}^{\mathrm{TM}}$ mode.

Acknowledgment. This work was supported by the Kyungnam University Research Fund, 2005.

\section{References and Notes}

1. (a) Hu, J.; Odom. T. W; Lieber. C. M. Acc. Chen. Res. 1999, 32 , 435. (b) Duan, X.; Huang, Y.; Cui, Y.; Wang, J.; Lieber, C. M. Nature 2001, 409, 66. (c) Poncharal, P.; Wang, Z. L.; Ugarte, D.; Heer, W. A. D. Science 1999, 283, 1513. (d) Cui, Y.; Lieber, C. M. Science 2001, 291, 851.

2. (a) Cui, Y.; Wei, Q.; Park, H.; Lieber, C. M. Science 2001, 293 , 1289. (b) Hahm, J.-I.; Lieber, C. M. Nano Left. 2004, 4. 51. (c) Zhang, D.; Li, C.; Liu, X.; Han, S.; Tang, T.; Zhou, C. Appl. Phys. Lett. 2003, 83, 1845. (d) Kolmakov, A.; Zhang, Y.; Cheng, G; Moskovits, M. Ad, Mater: 2003, 15,997.

3. (a) Chen, J.; Hamon, M. A.; Hu, H.; Chen, Y.; Rao, A. M.; Eklund, P. C.; Haddon, R. C. Science 1998, 282, 95. (b) Holzinger, M.; Vostrowsky, O.; Hirsch, A.; Hennrich, F.; Kappes, M.; Weiss, R.; Jellen, F. Angev. Chem, Int. Ed, 2001, 40, 4002. (c)
Georgakilas, V; Kordalos, K.; Pralo, M.; Guldi, D. M.; Holzinger, M.; Hirsch, A. J. Ant. Chem. Soc. 2002, 124, 760. (d) Bahr, J. L.; Yang, J.; Kosynkin, D. V.; Bronikowski, M. J.; Smalley, R. E.; Tour, J. M. J. Ant. Chemi. Soc. 2001, $J 23,6536$. (e) Yoon, K. R.; Kim, W.-J.; Choi, I. S. Macromol. Chent. Phys, 2004, 205, 1218 (I) Lee, Y.-W.; Kang, S. M.; Yoon, K. R.; Hong, S.-P.; Yu, B.-C.; Chi, Y. S.; Paik, H.-j.; Yun, W. S.; Choi, I. S. Macromol. Res. $2005,13,356$

4. (a) Sun, X. H.; Wang, S. D.; Wong, N. B.; Ma, D. D. D.; Lee, S. T.; Teo, B. K. Inorg. Chem. 2003, 42, 2398. (b) Duan, X.; Huang. Y.; Lieber, C. M. Nano. Lett, 2002, 2, 487, (c) Cui, Y,; Zhong, Z, Wang, D.; Wang, W. U.; Lieber, C. M. Namo Lett. 2003, 3, 149. (d) Wang, Y.; Tang, Z.; Tan, S.; Kotov, N. A. Nano Letf. 2005, 5, 243. (e) Hanrath, T.; Korgel, B. A. J. Am. Chem. Soc. 2004, 126, 15466.

5. (a) Zhang, Y. F,; Tang, Y. H.; Wang, N.; Yu, D. P.; Lee, C. S.; Bello, I.; Lee, S. T, Appl. Phys. Lett, 1998, 72, 1835. (b) Morales, A. M.; Lieber, C. M. Science 1998, 279, 208. (c) Yu, D. P; Bai, Z. G.; Ding, Y.; Hang, Q. L.; Zhang, H. Z.; Wang, J. J.; Zou, Y. H.; Qian, W.; Xiong, G. C.; Zhou, H. T.; Feng, S. Q. Appl. Phys. Lett. 1998, 72, 3458. (d) Wang. N.; Tang. Y. H.; Zhang, Y. F.; Yu. D. P.; Lee, C. S.; Bello, I.; Lee, S. T. Chem. Phys. Lett, 1998, 28,3, 368. (e) Cui, Y.; Lauhon, L. J.; Gudikser, M. S.; Wang, J.; Lieber, C. M. Appl. Phys. Lett, 2001, 78, 2214.

6. Buriak, J. M. Chem. Rev, 2002, 102, 1271.

7. Chi, Y. S.; Lee, J. K.; Lee, K.-B.; Kim, D. J.; Choi, I. S. Bull. Korean Chem. Soc. 2005. 26.361.

8. (a) Wasserman, S. R.; Tao, Y.-T.; Whitesides, G. M. Langmuir 1989, 5, 1074, (b) Lee, K.-B.; Kim, Y.; Choi, I, S. Bull. Korean Chem, Soc, 2003, 24, 161. (c) Lee, K.-B,; Kim, D. J.; Yoon, K. R.; Kim, Y.; Choi, I. S. Korean J. Chem. Eng. 2003, 20, 956. (d) Park. J. P.; Lee, S. J.; Park, T. J.; Lee, K.-B.; Choi, I. S.; Lee. S. Y.; Kim, M.-G; Chung, B. H. Biotechnol. Bioprocess Eng. 2004, 9.137.

9. (a) Lee, K.-B.; Kim, D. J.; Lee, Z.-W.; Woo, S. I.; Choi, I. S. Langmuir 2004, 20, 2531. (b) Lee, Z-.W.; Lee, K.-B.; Hong, J.-H.; Kim, J.-H.; Choi, I.; Choi, J. S. Chem. Lett, $2005,34,648$.

10. (a) Caswell, K. K.; Wilson, J. N.; Bunz, U. H. F.; Murphy, C. J. $J$. An. Chen. Soc. 2003, 125, 13914. (c) Salem, A. K.; Chen, M.; Hayden. J.; Leong. K. W.; Searson, P. C. Nano Left. 2004, 4, II 63.

11. Lee, K.-B.; Yoon, K. R.; Woo, S. I.; Choi, I. S. J. Pharm. Sci. $2003,92,933$.

12. The images shown in Figure 3 are aggregates of $\mathrm{SiNW}_{\mathrm{s}}$ and we realized that it was practically difficult to disperse $\mathrm{SiNWs}$ in aqueous medium even after modifying $\mathrm{SiNW}$ with biotin. The synthesis of water-soluble SiNWs is being investigated as an alternative approach. 